\title{
Evaluating Understanding of Popular Press Reports of Health Research
}

\author{
William H. Yeaton, PhD \\ Dawn Smith, MD \\ Karen Rogers, BA
}

This research assessed the ability of a sample of persons on a college campus to understand media reports of health research. Three or four articles on each of five contemporary health topics (dietary cholesterol and heart disease, treatment for breast cancer, starch blockers, drug treatment for heart disease, test tube skin) were selected from widely circulated newspapers (e.g., New York Times) and magazines (e.g., Newsweek). A sample of 144 college students responded to content-based and applicationbased questions derived from photocopies of these popular press articles. The overall rate of reader misunderstanding approached $40 \%$ and generally fell beween one third and one half for each of 16 articles representing five health topics. Several strengths and weaknesses of the research are considered as they relate to the accuracy of estimated error rates and to the generality of study findings. The implications of these findings for other areas of health (e.g., AIDS risk factor research) are also discussed.

\section{INTRODUCTION}

Our collective and individual well being increasingly depend on the production and transfer of information. The availability of personal health information is especially critical as scientific research identifies the causes of new diseases. In fact, Americans can now minimize their risks for 9 of the 10 leading causes of death by altering lifestyle and by changing individual behaviors, ${ }^{1}$ provided they possess pertinent health information and sufficient motivation to use it.

In response to the demand for health information, media sources that provide relevant medical news and advice have proliferated..$^{2.3}$ Television and radio have developed syndicated programs, and networks sometimes support reporters who specialize in science or hire physicians who translate and disseminate medical research findings. Several newspapers (e.g., New York Times, Detroit Free Press) in large metropolitan areas regularly devote sections to health topics and health

The authors would like to thank Pam Adelman and Amiram Vinokur for their helpful comments on earlier drafts of the paper.

The authors are with the Institute for Social Research. University of Michigan.

Address reprint requests to William $\mathrm{H}$. Yeaton, University of Michigan, Ann Arbor, MI 48106. 
research. Magazines such as Scientific American, Science News, and Discovery, though aimed at science professionals, have begun to acquire a more diverse readership. Magazines devoted to health topics typically translate medical research reports into lay terms, and more general periodicals (e.g., Newsweek, Time) routinely cover medical issues, ${ }^{4}$ sometimes offering behavioral advice.

Media coverage of AIDS is the latest case in a long list of health topics including tobacco and alcohol abuse, cancer screening, and cardiovascular fitness now widely reported by the popular press. Such coverage is a potentially efficient element in the health education of a whole society. For example, when a Swedish newspaper undertook a one-year health information campaign focused on smoking cessation, diet modification, and increased exercise, follow-up studies showed that $97 \%$ of the population were aware of the program..$^{5}$

Despite the wealth of available health information and society's general awareness of it from reading popular press reports, relatively little is known about the ability of lay consumers to understand and to utilize health information in intelligent ways. In addition, individual decision-making may be complicated by the complexity of issues and the open disagreement of experts. As a result, some women defer mammography, worried that it will cause cancer. Other persons avoid donating blood, fearing that they could contract AIDS in the process.

Collective decision making may likewise be deficient. For example, media reports may directly affect important policy changes. Norman identified what he termed "media disease" in the case of publicity about urea-formaldehyde foam (UFF) insulation. Before media reports appeared, families in UFF insulated homes reported no increased symptoms compared to families in non-UFF homes, though after the blitz of media stories such families reported $30-300 \%$ more symptoms. Studies eventually demonstrated no significant difference in formaldehyde levels between homes with such insulation and those without. However, bans of UFF insulation have been continued in Canada and in the United States.

In some instances, mistakes in individual and collective decision making may be attributable to the poor quality of the translation of original research reports to a particular secondary source. When scientists publish their research or announce their recommendations based on available scientific findings, the media interpret these findings for the lay public. Unfortunately, writers' ability to communicate effectively may be constrained by several factors including their own understanding of statistics and sampling, relevant scientific principles germane to the research, ${ }^{7}$ their writing technique, ${ }^{8}$ the differing purposes of scientists and writers, ${ }^{9}$ and the modest space allotted to the topic by their editors. ${ }^{10}$

Although many previous studies have focused on those factors cited above, far fewer investigations have focused on the interpretation of health information by lay consumers. ${ }^{11}$ The present study addresses the quality and composition of media reports only indirectly (see Berry ${ }^{12}$ and Tankard and Ryan ${ }^{13}$ for more direct approaches). Instead, it focuses on the ability of lay readers to understand media reports of health research. This basic understanding is one of a host of factors vital to improved lay decision making ${ }^{1+}$ and exerts considerable influence on future health care choices. 
In this research study, we first identified five contemporary health topics judged to be important and of potential interest to a sample of college-educated laypersons. Written questionnaires evaluated the degree of understanding shown by college students who read a single, secondary article.

\section{METHODS}

\section{Participants and Sites}

This study was conducted at five sites on the University of Michigan campus where students naturally congregate. Eighty two percent of the respondents were between 18 and 25 years of age; only $7 \%$ were 30 or older. Sites were chosen for which there would be sufficient waiting time to allow each participant to complete a questionnaire (e.g., the waiting room of the health center). In addition, students in a general marketing class completed questionnaires after receiving minimal instructions from one of the researchers. A total of 144 students participated in this study.

\section{Choice of Secondary Articles}

Several criteria guided the selection of secondary articles. First, each secondary article discussed an important health issue and appeared in a widely circulated newspaper or magazine. Second, selected articles had to represent a variety of basic and applied health topics judged likely to hold the interest of a diverse sample of college students. Third, only those secondary articles were chosen that focused on information presented in a single, identifiable primary article. Fourth, the focus of discussion within each of the five health topics was a single, published health research article. Then, we located several secondary reports that had been based on individual primary articles.

Based on these criteria, we chose secondary articles that addressed the following issues (the number of secondary articles appears in parentheses): surgical alternatives for breast cancer (3), drug treatment for congestive heart disease, (3), use of starch blockers for weight reduction (3), dietary cholesterol and heart disease (4), and cultured skin transplants for burns (3). These secondary articles were taken from several newspapers (New York Times, Detroit Free Press, Ann Arbor News), news magazines (Newsweek, Time), and science magazines (Discover, Omni, Science Digest, Science News). It was assumed that these secondary sources of health information shared generally similar levels of reading difficulty.

\section{Questionnaire Development and Format}

The major purpose of this research was to assess the frequency and types of misunderstandings made by laypersons who were asked to read popular press reports of research on important health topics. Errors were based on participant 
responses after reading secondary articles. Our intent was not to directly assess the quality of the "translation" between each primary article and its respective set of secondary articles. However, two of the authors checked the accuracy of statements in secondary articles by referring to relevant data published in the primary article. By comparing each primary article with its set of secondary articles, we were able to identify potential areas of misunderstanding, including inferences that might be overgeneralized, incorrectly stated, or inadequately qualified, as well as more straightforward knowledge of facts and principles.

On page one of each questionnaire participants were asked to provide background information (age, sex) and to report any personal or immediate family experience with the treatment in question. A copy of the secondary article appeared on page two. All references to its publication source were removed. Participants were instructed to read the article and to answer questions on the following pages without referring back to the secondary article.

A variety of question formats were used including true-false, multiple choice. and yes/no. For example, one of the multiple choice questions, designed to test understanding of the article entitled "Study finds digitalis helps half the patients," asked respondents to choose the correct response from four options (the correct answer is " 1 "): The study group included: (1) men of many ages with different types of congestive heart failure, (2) only patients whose condition was thought to result from arterial or muscular deterioration, (3) only patients whose hearts had a "third sound," and (4) only patients with diseased heart valves.

We designed individual questions to assess several dimensions of reader comprehension of each health report: general understanding of each research study, recall of the study methods used by the investigators to reach conclusions, recall of important details which qualified the circumstances in which the reported finding would apply, and the capacity to make correct inferences from the information in each secondary article. On two topics a Likert scale was used to assess a reader's behavioral inclination to accept or reject a given treatment when placed in a hypothetical choice situation.

On each questionnaire a single question was designed to test whether a participant had carefully read the secondary article. In this case, incorrect options presented to the respondent were entirely unrelated to the content of the article. These questions were not utilized in the calculation of error rates. Incorrect responses to such questions enabled us to identify participants who responded in a careless manner and to omit their data.

Questions fell into two general categories: those that tested readers' understanding of content, and those that tested their ability to recognize correct and incorrect application of study results. Content questions tested recall of important facts, concepts, and principles as well as knowledge of the precise nature, rationale, and mechanism of treatment. For example, in the set of secondary articles on starch blockers, we tested participants' recall of one article's explanation for the ineffectiveness of starch blockers with the question, "Starch blockers are hypothesized not to be effective in humans because they do not block all of the body's digestive enzymes which work on starch." (The correct answer was "true.") Applicability questions tested knowledge of the conditions of generality-those samples of persons, time periods, and circumstances in which 
the study had been conducted and for which inferences were likely to hold. For example, the correct response ("false") to the question, "The study shows that all Americans should lower their dietary cholesterol in order to reduce their risk of heart attack," was designed to test whether respondents would overgeneralize study findings to persons not in the reported research (in this case, middle-aged men participated in the research). Correct answers to applicability questions also required knowledge of success rates and possible side effects since these results would represent important qualifiers to the overall study conclusions.

For each of the five health topics, we developed a different questionnaire, though a few questions were identical across topics. Within each health topic, an identical set of questions appeared on the questionnaire for each secondary article along with a small number of questions that probed unique aspects of a particular secondary article.

A preliminary version of the questionnaire for one health topic was pilot tested on an undergraduate class in research methods at the University of Michigan. Based on feedback from class members, several changes in wording and format were made which improved the clarity of questions for each health topic.

\section{Questionnaire Distribution}

A poster detailing the purpose and participation requirements of the study accompanied questionnaires at each campus site. All questions were designed to be answered without researcher assistance. Each questionnaire was placed in an individual envelope, and general directions provided on page one instructed each participant to "Please read the article to which this sheet is attached. Take about as much time as you normally might in reading a newspaper or magazine article." During a second pilot test, one of the researchers unobtrusively observed students picking up, completing, and returning their questionnaires to a designated pick up point. No difficulties or protocol irregularities (e.g., turning back to the article to answer questions) were noted.

Initially, a small set of 10-20 questionnaires containing an equal number of the five topics was placed at every site. Using a table of random numbers, the set of questionnaires was randomly ordered within each of the sites. A researcher checked each site every third day to replenish each set or to transfer questionnaires from a lower completion rate site to a higher completion rate site. On each visit, University staff in the general area indicated that there had been no irregularities, and direct observation of subjects revealed little variation in study protocol.

\section{Data Compilation}

Only those questionnaires were retained for which one and only one response was made to each question. In addition, questionnaires were excluded $(n=6)$ if a participant responded incorrectly to the single question on each questionnaire that demanded minimal attention since this failure suggsted that motivation or attentiveness had been lacking. 


\section{RESULTS}

Table 1 contains descriptive information from 144 questionnaires completed at five sites. While there was considerable variation in the number of completed questionnaires at each of the five sites (range $=16-53$ ), more than a third of the questionnaires were completed at the health services center. For the five health topics, the mean age of respondents varied from 22-24 years, and the percent of males and females was consistently near $50 \%$.

The percent of incorrect responses for each of the five health topics and a mean percent averaged across all five topics are reported in Table 2 . This table also provides a breakdown of error rates for each of the 16 secondary articles.

Based on the total number of correct and incorrect responses to individual questions, the overall percent of errors was 39 , while the range across the five topics was $34-44 \%$. The percent of errors by health topic was:

cultured skin transplants, $38 \%$

dietary cholesterol and heart disease, $43 \%$

surgical treatments for breast cancer, $37 \%$

drug treatment for congestive heart disease, $34 \%$

starch blockers, $44 \%$

Each of these five percents and the overall percent of errors were statistically significant ( $p<.01$, one-tailed test). None of the paired contrasts between topic totals was significant $(p<.01$, two-tailed test).

When errors were aggregated within each of the 16 secondary articles used in this research, the range was $31-47 \%$. The percent of errors in each of the 16

Table 1. Descriptive Information $(N=144)$

\begin{tabular}{|c|c|c|c|c|}
\hline \multicolumn{3}{|c|}{ By location } & \multicolumn{2}{|c|}{$\begin{array}{l}\text { Questionnaires } \\
\text { Completed }\left(N=143^{4}\right)\end{array}$} \\
\hline Site 1 (health service & & & & \\
\hline Site 2 (counseling) & & & & \\
\hline Site 3 (counseling) & & & & \\
\hline Site 4 (career planni & and placem & $n t)$ & & \\
\hline Site 5 (classroom) & & & & \\
\hline By health topict & $\begin{array}{l}\text { Secondary } \\
\text { articles }\end{array}$ & $\begin{array}{c}\text { Number with personal } \\
\text { or family } \\
\text { experience }\end{array}$ & Female $(\%)$ & Mean age \\
\hline Test tube skin & 3 & 8 & 48 & 22 \\
\hline $\begin{array}{l}\text { Dietary cholesterol } \\
\text { and heart disease }\end{array}$ & 4 & 23 & 44 & 24 \\
\hline $\begin{array}{l}\text { Breast cancer } \\
\text { treatment }\end{array}$ & 3 & 10 & 55 & 23 \\
\hline $\begin{array}{l}\text { Drug treatment for } \\
\text { heart disease }\end{array}$ & 3 & 7 & 47 & 23 \\
\hline Starch blockers & 3 & 2 & 50 & 22 \\
\hline
\end{tabular}

"The site of one completed questionnaire was not determinable.

b Based on sample before unusable questionnaires were excluded. 
Table 2. Percent Incorrect Responses by Topic and Secondary Article ${ }^{\mathrm{a} . b}$

\begin{tabular}{|c|c|c|c|c|c|c|}
\hline & \multirow[b]{2}{*}{ Topic } & \multicolumn{4}{|c|}{ Secondary Article } & \multirow[b]{2}{*}{ Total } \\
\hline & & 1 & 2 & 3 & 4 & \\
\hline \multirow[t]{2}{*}{1} & Test tube skin & $44 \%$ & $38 \%$ & $\mathbf{3 1} \%$ & & $38 \%$ \\
\hline & & $\begin{array}{c}32 / 72 \\
(n=9)\end{array}$ & $\begin{array}{c}24 / 64 \\
(n=8)\end{array}$ & $\begin{array}{c}25 / 80 \\
(n=10)\end{array}$ & - & $\begin{array}{c}81 / 216 \\
(n=27)\end{array}$ \\
\hline \multirow[t]{2}{*}{2} & Dietary cholesterol & $42 \%$ & $45 \%$ & $41 \%$ & $44 \%$ & $43 \%$ \\
\hline & and heart disease & $\begin{array}{c}34 / 80 \\
(n=10)\end{array}$ & $\begin{array}{c}25 / 56 \\
(n=7)\end{array}$ & $\begin{array}{c}26 / 64 \\
(n=8)\end{array}$ & $\begin{array}{c}28 / 64 \\
(n=8)\end{array}$ & $\begin{array}{c}113 / 264 \\
(n=33)\end{array}$ \\
\hline \multirow[t]{2}{*}{3} & Breast cancer & $\mathbf{3 1} \%$ & $45 \%$ & $32 \%$ & & $37 \%$ \\
\hline & treatment & $\begin{array}{c}22 / 70 \\
(n=10)\end{array}$ & $\begin{array}{c}34 / 75 \\
(n=11)\end{array}$ & $\begin{array}{c}21 / 65 \\
(n=9)\end{array}$ & - & $\begin{array}{c}77 / 210 \\
(n=30)\end{array}$ \\
\hline \multirow[t]{2}{*}{4} & Drug treatment & $32 \%$ & $32 \%$ & $\mathbf{3 8 \%}$ & & $34 \%$ \\
\hline & for heart disease & $\begin{array}{c}28 / 88 \\
(n=11)\end{array}$ & $\begin{array}{c}23 / 72 \\
(n=9)\end{array}$ & $\begin{array}{c}39 / 104 \\
(n=13)\end{array}$ & - & $\begin{array}{c}90 / 264 \\
(n=33)\end{array}$ \\
\hline \multirow[t]{2}{*}{5} & Starch blockers & $39 \%$ & $47 \%$ & $47 \%$ & & $44 \%$ \\
\hline & & $\begin{array}{c}19 / 49 \\
(n=7)\end{array}$ & $\begin{array}{c}23 / 49 \\
(n=7)\end{array}$ & $\begin{array}{c}23 / 249 \\
(n=7)\end{array}$ & - & $\begin{array}{c}65 / 147 \\
(n=21)\end{array}$ \\
\hline \multicolumn{2}{|c|}{ Overall Total } & & & & & $\begin{array}{c}39 \% \\
426 / 1101 \\
(n=144)\end{array}$ \\
\hline
\end{tabular}

a Proportions for each of the 16 secondary articles were significantly different than zero ( $p<.01$, one-tailed test).

${ }^{\mathrm{h}}$ None of the paired contrasts between topic totals was significant $(p<.01$, two-tailed test). None of the paired contrasts between the three or four secondary articles within each topic was significant $(p<.01$, two-tailed test).

topics was statistically significant ( $p<.01$, two-tailed tests). None of the paired contrasts between the three or four secondary articles within each topic was significant ( $p<.01$, two-tailed test). Thus, whether reported as an overall average or as an average by topic or by article, the proportion of errors was significantly different from zero but quite homogeneous, generally falling between one third and one half.

Though not reported in Table 2, further breakdowns were conducted by individual questions in different formats (e.g., true/false, multiple choice) and in different types (e.g., content-based, application-based), and for questions that were either the same or different in each secondary article within a particular health topic. These results reflected a much wider range in the percent of incorrect responses $(0-87 \%)$ to individual questions within a particular secondary article and health topic. Questions which were identical across secondary articles generally resulted in more incorrect responses (41.6\% errors) than those questions which differed across articles (30.6\% errors). As might be expected, respondents had greater difficulty with applicability-based questions than with content-based questions. This latter pattern held for four of the five topics and was magnified for questions that were identical across topics.

For two topics, we assessed the behavioral inclinations of respondents after reading their secondary article and completing their questionnaire. For the dietary cholesterol and heart disease topic, we asked participants "How likely are 
you to decrease your cholesterol intake as a result of reading this article?" The means for those participants who responded to this question on each of the four secondary articles were $3.6,2.1,2.8$. and 3.6 , where $1=$ very likely and $7=$ very unlikely. For the breast cancer treatment topic, we asked participants "How likely would you be to encourage a relative eligible for lumpectomy treatment to have a lumpectomy rather than a mastectomy?" The mean responses for the three secondary articles were $3.8,4.2$, and 5.8 , where $1=$ very likely and $10=$ very unlikely.

\section{DISCUSSION}

This research evaluated the ability of college educated laypersons to respond correctly to a series of questions immediately after reading an article published in the popular media that had reported health research results. The overall percent of errors approached $40 \%$, falling between one third and one half for each of the five topics. Similarly, error rates for the 16 secondary articles were also quite homogeneous, falling consistently between $30 \%$ and $50 \%$.

\section{Implications of the Findings}

The error rates found in this research are a source of considerable concern from a number of vantage points. First, if one assumes that an adequate understanding of health research should be an important foundation on which to base future health decisions, it is obvious that subsequent decision making will be flawed. Second, these errors are likely to have both direct and indirect implications. For example, there will be indirect effects if one's inability to accurately recall health information leads to further distortion when related information is processed at a subsequent point in time. In terms of direct effects, high rates of misunderstanding in application-based questions are bothersome since this deficiency suggests that consumers may misjudge the applicability of research findings to their own, unique circumstances. Third, an error rate near $40 \%$ is unacceptably high, approaching chance levels. In a normative sense, the error rates are in the range of those reported in studies testing laypersons' understanding of scientific issues. ${ }^{15}$

These high error rates are particularly troublesome since numerous aspects of this research would lead one to conclude that our results represent an overestimate of consumer ability to understand health research in popular media reports. The five topics represented a variety of health issues likely to be familiar to this audience. Thus, the concepts on which many of the questions were based are not likely to pose problems because of their novelty. Furthermore, the articles were based on potentially interesting, contemporary health research results, were short (copies were made on a single $81 / 2$ by 11 -in. page), and were taken from newspapers and magazines, sources that are likely to be relatively easy reading for a college student sample admitted to a major university. Questionnaires required little time to complete (approximately 5-10 minutes), and recall was tested without any delay. A moderate level of motivation can be assumed since all participants were volunteers and were aware that their responses would 
subsequently be scrutinized more closely. Informal and occasional observation by one of the researchers suggested that respondents worked diligently to complete their questionnaires. Finally, a question included in each questionnaire identified potentially inattentive respondents, and their results were excluded.

\section{Generalizability of the Findings}

One should be cautious in generalizing from the relatively small number of media sources and articles on which these results are based. The health reports used in this research were taken from three newspapers and six science and news magazines. A modest sample size of 144 college students read a total of 16 secondary articles on five different health topics. Replication of these results with other health topics, secondary articles, and media sources by other consumers of health information would enhance this study's generalizability.

In addition, one should note carefully those conclusions which were neither made nor possible. No attempt was made to compare the ability of consumers to reach correct inferences within specific sources (Newsweek, Time), in different kinds of sources (e.g., newspapers or magazines), in sources geared to general or more educated audiences (e.g., Newsweek or Science News), or on topics of different complexity (e.g., starch blockers or breast cancer). General conclusions based on differing error rates for such comparisons would require a much larger and more exhaustive sample of articles.

However, several aspects of this research suggest that the findings do apply more generally. Secondary articles were photocopied directly from actual reports in the popular media; they were not facsimiles rewritten by research staff. Articles were chosen to represent important and potentially interesting, contemporary topics. Articles were not selected based on our impression of their reporting quality. All secondary articles identified in our search that discussed a designated primary research article were included in this research.

In addition, the relatively uniform rate of errors across different health topics and secondary articles is consistent with a conclusion that consumer misunderstanding of health reports in popular media is pervasive. The fact that articles were randomly allocated to respondents makes it likely that participant characteristics (e.g., reading levels or motivational factors) contributed little to the differences in error rates between secondary articles within topics. By choosing several secondary reports of the same primary article for each health topic, we were able to minimize the possibility of reader misunderstanding due to the style, emphasis, or clarity of a particular secondary source. Finally, the use of identical questions for different secondary articles which contained similar information strengthens the conclusion that a consistent deficiency exists in consumers' ability to correctly interpret secondary reports of health research.

\section{Statistical Conclusion Validity}

It is also important to evaluate the statistical underpinnings of the pattern of these results, namely that the error rates found in each of the topics and articles are significantly different from zero but similar in magnitude. ${ }^{16}$ First, to protect 
against experiment-wise error stemming from the large number of statistical tests conducted, ${ }^{17}$ an alpha of .01 was used in each test. Since the percent of errors in the 16 articles was consistently over $30 \%$ (the smallest error rate was .31 , and its standard deviation was .05), the chances of a Type I error are quite low.

Second, was there adequate statistical power to protect against Type II error? ${ }^{18}$ There are two types of cases to consider, those that test the statistical significance of error rates relative to a zero rate and those that test differences between results within articles and topics. In the first case, if one assumes the 144 participants are equally distributed among each of the 16 cells in the research design ( $n=9$ per cell), an error rate of $39 \%$, an alpha of .01 , and a one-tailed test of statistical significance (to test that error rates are greater than zero), the statistical power is approximately .73. A more liberal assumption that sample size should be based on the number of answers to questions given by the participants in each cell (the average is 69) yields power $=.99$.

In the second case, to test for differences between articles within topics, assuming an average difference of $10 \%$ ( 35 vs. $45 \%$ ), a sample size of nine, an alpha of .01 , and a two-tailed test of significance (since one could not predict which article would have the higher error rate), the statistical power is .02. Using 69 as a more liberal assumption for sample size yields a power of .08. Assuming that a participant is the correct unit of analysis to test for differences between articles, nearly 600 persons per article (approximately 10,000 total participants) would be required to achieve a power of .80 . Therefore, based on purely statistical criteria, conclusions related to similar error rates between articles are a great deal more tenuous than those related to the statistical significance of individual article error rates. For practical purposes, however, the fact that the difference between the largest and smallest percent of errors in the 16 articles was $16 \%$ suggests that lack of reader understanding is a problem of similar magnitude in secondary research reports.

\section{Future Directions}

This research did not probe the impact of secondary reports on changes in actual respondent behavior. Instead it assessed understanding and knowledge, early steps in the behavior change process (see Flay, ${ }^{19}$ p. 68 , for an example of an integrative model of attitude and behavior change). However, the data we obtained on behavioral inclinations for two of the topies, dietary control of cholesterol and breast cancer treatment, indicated that participants were favorably inclined to the treatment and its reported effectiveness as it had been described in the secondary article. In future studies, one could evaluate the effect of popular reports of health information on behavioral inclination by assesssing participants' inclinations both before and after exposure. Given the predictive power of stated inclinations on future behavior, ${ }^{20}$ this approach would enable one to assess the role of correct and incorrect interpretation of health information on future behavior and to examine the factors that determine appropriate and inappropriate actions in response to media reports.

Lay ability to understand health information is imperative for those health topics in which the cost of consumer misunderstanding is high. For example, in 
educating sexually active young adults about the risks of contracting AIDS, ${ }^{21}$ failure to alter behavioral risk factors cannot be attributed to the lack of media coverage of AIDS research. Instead, it may be due to a low perceived probability of contracting AIDS, the presence of immediate rewards, or to an inadequate understanding of the risk factors involved. ${ }^{15.22}$

A recent survey of high school students indicated that newspapers and magazines are popular sources of information on AIDS. ${ }^{23}$ In fact, students who reported that they obtained AIDS information from these two sources also ranked relatively high in AIDS knowledge. However, their absolute knowledge scores were low ( 9.08 and 8.75 for magazines and newspapers, respectively, out of a possible score of 19). This research does not, however, allow one to determine the extent to which media reports of varying quality actually contribute to low levels of student understanding of AIDS risk. Without knowing the degree to which information is incorrectly understood by consumers, it will not be possible to intelligently target improved approaches for the dissemination of vital health information. ${ }^{24}$

Our research indicates that many readers do not adequately understand reports of health research at the time of initial exposure. Unfortuantely, this finding is all too reasonable in light of minimal feedback obtained by science writers before a media report is published. Written or verbal commentary from an editor cannot be expected to reflect the degree to which an article will be understood by a lay audience. Rather, editorial feedback is usually intended to address the potential impact of an article on readership. We recommend that writers seek feedback from several lay readers before an article is published, thus allowing clarification of results that might be misunderstood or misapplied. More fundamentally, it may also be necessary to alter basic dimensions of media reports such as the style of presentation (e.g., inclusion of a brief paragraph summarizing the findings) to enhance consumer understanding of health research reports.

\section{References}

1. National Center for Health Statistics: Health U.S., 1987. Rockville, MD, Public Health Services Publication 88-1232, 1988.

2. Broad WJ: Science magazines: The second wave rolls in. Science 215:272-273, 1982.

3. Culliton BJ: Science sections in U.S. newspapers increased dramatically in past two years. Science 235:429, 1987.

4. Winkler JD, Kanouse DE, Brodsley L, Brook RH: Popular press coverage of eight National Institutes of Health Consensus Development topics. Journal of the American Medical Association 255:1323-1327, 1986.

5. Malgrem S, Andersson G.: Who were reached by and participated in a one year newspaper health information campaign? Scandinavian Journal of Social Medicine 14:133-140, 1986.

6. Norman GR: Science, public policy, and media disease. Canadian Medical Association Journal 134:719-720, 1986.

7. Borman SC: Communication accuracy in magazine science reporting. Journalism Quarterly 55:345-346, 1978.

8. Bostian LR: How active, passive, and nominal styles affect readability of science writing. Journalism Quarterly 60:635-640, 1983. 
9. McCall RB: Science and the press. Like oil and water? American Psychologist 43:8794. 1988.

10. Winsten J: Science and the media: The boundaries of truth. Health Affairs 4:5-23, 1985.

11. Grunig JE: Three stopping experiments on the communication of science. Journalism Quarterly 51:387-399, 1974.

12. Berry FC: A study of accuracy in local news stories of three dailies. Journalism Quarterly 44:482-490, 1967.

13. Tankard JW, Ryan M: News source perceptions of accuracy of science coverage. Journalism Quarterly 51:219-225, 1974.

14. Nisbett R, Ross L: Human Inference: Strategies and Shortcomings of Social Judgment. Englewood Cliffs, NJ, Prentice-Hall, 1980.

15. DiClemente RJ, Zorn J, Temoshok L: Adolescents and AIDS: A survey of knowledge. attitudes, and beliefs about AIDS in San Francisco. American Journal of Public Health 76:1443-1445, 1986.

16. Cook TD, Campbell DT: Quasi-Experimentation: Design and Analysis Issues for Field Settings. Chicago. Rand McNally, 1979.

17. Kirk RE: Experimental Design: Procedures for the Behavioral Sciences. Belmont, CA, Brooks/Cole, 1968.

18. Cohen J: Statistical Power Analysis for the Behavioral Sciences. New York, Academic Press, 1977.

19. Flay BR: On improving the chances of mass media health promotion programs causing meaningful changes in behavior, in M Meyer (ed): Communication Research and Broadcasting Vol. 5, 1981.

20. Ajzen I, Fishbein M: Understanding Attitudes and Predicting Social Behavior. Englewood Cliffs, NJ, Prentice-Hall, 1980.

21. Koop CE: Teaching children about AIDS. Issues in Science and Technology Fall:67$70,1987$.

22. DiClemente RJ, Boyer CB, Morales ES: Minorities and AIDS: Knowledge, attitudes, and misconceptions among Black and Latino adolescents. American Journal of Public Health 78:55-57, 1988.

23. Price JH, Desmond S, Kukulka G: High school students' perceptions and misperceptions of AIDS. Journal of School Health 55:107-109, 1985.

24. Flay BR, Cook TD: Evaluation of mass media campaigns, in Rice RE, \& Paisley WJ, (eds): Public Communications Campaigns. Beverly Hills, Sage, 1985. 